\title{
Valoración del estado nutricional de pacientes en hemodiálisis del Centro de Hemodiálisis SERSALUD Amazonia E.I.R.L. Iquitos, 2016
}

\author{
Assessment of nutritional status of hemodialysis patients Hemodialysis Center SERSALUD Amazon \\ E.I.R.L. Iquitos, 2016
}

Mercy Lizeth Becerra Ortiz*, Elisa Romy Rodríguez López²

\begin{abstract}
RESUMEN
Objetivo: Valorar el estado nutricional de los pacientes en hemodiálisis. Metodología: Enfoque cuantitativo, de diseño no experimental, de corte transversal y de tipo descriptivo. La muestra estuvo conformada por 100 pacientes entre las edades de 20 y 80 años, el 60\% fueron hombres y 40\% mujeres. Se utilizó los instrumentos de Valoración Global Subjetiva (VGS), Valoración Global Objetiva (VGO) y recordatorio de 24 horas. Resultados: Por el Índice de Masa Corporal, el 54\% normal y el 19\% desnutrición leve y moderada. Respecto a la circunferencia braquial (CB), el 93\% se encuentra adecuado. Por la circunferencia muscular del brazo (CMB), en el $45 \%$ se encuentra adecuada reserva proteica. El Pliegue Cutáneo Tricipital (PTC), el 27\% en adecuada reserva calórica, con desnutrición leve y moderada el 27\% y 24\%, respectivamente. Por albumina sérica, el $76 \%$ con desnutrición leve. La transferrina sérica, el 60\% presentó desnutrición moderada y recuento de linfocitos totales, el 32\% en desnutrición leve. El 19\% mostró signos leves de ascitis o edemas. Y en cuanto por recordatorio de 24 horas, el 47\% presentó desnutrición moderada por la ingesta proteica, el 96\% se encuentra inadecuado por la ingesta calórica. Conclusiones: VGS se encontró que el 99\% tiene desnutrición leve, mientras que el 25\% desnutrición moderada y 75\% desnutrición leve según VGO.
\end{abstract}

Palabras clave: Estado nutricional, antropométricos, bioquímicos, dietéticos, hemodiálisis.

\begin{abstract}
Objective: To assess the nutritional status of patients on hemodialysis. Methodology: quantitative approach, nonexperimental, cross-sectional and descriptive. The sample consisted of 100 patients between the ages of 20-80 years, 60\% were men and 40\% women. instruments Subjective Global Assessment (SGA), Global Assessment Objective (VGO) and 24-hour recall was used. Results: By body mass index, 54\% normal and 19\% mild and moderate malnutrition. Regarding arm circumference (CB), 93\% is appropriate. By muscular arm circumference (CMB), 45\% is adequate protein reserve. The triceps skinfold (PTC), 27\% in adequate caloric reserve, with mild and moderate malnutrition $27 \%$ and $24 \%$, respectively. By serum albumin, $76 \%$ with mild malnutrition. Serum transferrin, 60\% had moderate malnutrition, and total lymphocyte count, 32\% in mild malnutrition. 19\% showed slight signs of ascites or edema. And as for 24 -hour recall, $47 \%$ had moderate malnutrition protein intake, $96 \%$ are unsuitable for caloric intake. Conclusions: By the VGS found that 99\% have mild malnutrition, while 25\% moderate and 75\% mild malnutrition according VGO.
\end{abstract}

Keywords: Nutritional status, anthropometric, biochemicals, dietitians, hemodialysis.

\footnotetext{
${ }^{1}$ Licenciada en Nutrición Humana, Coordinadora de la Unidad de Nutrición y Dietas. Clínica Adventista Ana Stahl, Iquitos, Perú. ${ }^{2}$ Docente de la EP Nutrición Humana, Facultad de Ciencias de la Salud, Universidad Peruana Unión, Lima, Perú.
} 


\section{INTRODUCCIÓN}

El estado nutricional es el resultado del equilibrio entre la ingestión de alimentos y las necesidades nutricionales del individuo; que pueden intervenir diferentes conjuntos de interacciones de tipo biológico, como alguna patología, edad, cambios sensoriales, entre otras; psicológico, como depresión, saciedad, inapetencia; y a nivel social como económico y accesibilidad de los productos, entre otros (Secretaría de Salud, 2013).

Por otro lado, la Enfermedad Renal Crónica (ERC) o la Insuficiencia Renal Crónica (IRC) es el resultado de diversas enfermedades crónicodegenerativas entre las que destacan la diabetes mellitus y la hipertensión arterial, así como el de las complicaciones cardiovasculares que son la principal causa de muerte, fenómenos que ocurre de manera similar en todo el mundo y en el Perú, son las causas más frecuentes de ingreso a diálisis (Palacios et al. 2010).

De acuerdo con Jiménez et al. (2012), los pacientes con Insuficiencia Renal Crónica tienen alta prevalencia de desnutrición energética proteica. Entre las principales causas de la desnutrición están los cambios en el metabolismo energético, trastornos hormonales, anorexia, problemas gastrointestinales como diarreas, estreñimiento, náuseas, vómitos, constante inflamación y toxicidad urémica. Marcadores nutricionales tales como baja albúmina de suero y bajo Índice de Masa Corporal (IMC) se asocian con una mayor mortalidad en esta población.

De hecho, según el informe y Registro Español de Enfermedades Renales, de la Sociedad Española, la prevalencia de IRC continúa aumentando por encima de los 1100 pacientes por millón de población (pmp) mundial, siendo el $14 \%$ de los pacientes con IRC diabéticos y un 51.9\% que se realizaron trasplante renal. La mortalidad de la IRC se mantiene a años anteriores en torno a un 8\% anual, siendo en Hemodiálisis un $14.7 \%$ y Diálisis Peritoneal un 8.3\%. Por otro lado, en el Perú en el 2007 se reportó el $18.3 \%$ de desnutrición entre leve y moderada con una albúmina promedio de $3.48+/-0.89$ en pacientes en hemodiálisis (Sociedad Española de Nefrología, 2015).

Cabe mencionar que, en los últimos años, se ha considerado la importancia de un estado nutricional adecuado en el paciente hospitalizado, debido a que la desnutrición incrementa las complicaciones infecciosas y no infecciosas; la morbilidad y mortalidad aumenta los tiempos de internación hospitalaria y la etapa de recuperación y rehabilitación posthospitalaria acrecienta los costos en las instituciones de salud. Se conoce la existencia de varios factores implicados en el desarrollo de esta malnutrición, como son la disminución de la ingesta calórico-proteico, las pérdidas proteicas durante la diálisis y el incremento del catabolismo relacionado con la misma (Guerrero, 1999; García et al. 2000).

Yanowsky et al, (2015) realizaron un estudio en México, demostrando que la hipoalbuminemia y el DPE son muy frecuentes en estos tipos de pacientes. Asimismo, no existe asociación entre los niveles de albúmina sérica y la VGS. Asimismo, Espahbodi et al, en Iran (2014) concluyeron que la EGS es la mejor herramienta para evaluar el estado nutricional en pacientes en hemodiálisis. De la misma manera, Montalvo y Gómez (2007), en Lima, evidenciaron que la VGS ha sido de utilidad para valorar el estado nutricional de manera clínica. Además, Noralí (2013), en Santa Fe, se observó que el consumo de macro y micronutrientes, tanto energético y proteico es deficiente. Lo que se recomienda tener una evaluación más completa, para ayudar en su tratamiento y llevar una mejor calidad de vida. A parte de ello, García et al, (2000) en Sevilla, encontraron una alta prevalencia de malnutrición proteica, en un $70 \%$ de los pacientes, frente a la calórica en un 21\%. Se debe agregar que Cardoso y Pérez (2015) en Chiclayo, encontraron que la supervivencia de los pacientes que presentaron diabetes mellitus más el daño de órgano comparado con los que no la presentaron fue de $80 \%$ ( $\mathrm{P}<0.001)$.

Por otro lado, Yuste et al, (2013) en Madrid, observaron que no hay correlación estadística entre las modificaciones de masa magra y masa grasa con las modificaciones de parámetros nutricionales, llegando a la conclusión que la valoración del estado nutricional, mediante parámetros analíticos, no presenta relación con los parámetros de composición corporal ni con sus modificaciones. También, Bravo et al, (2010) en México, evidenciaron un incremento en la cantidad de masa grasa (MG) en la población estudiada. Por el contrario, no existió evidencia de desnutrición proteica. Asimismo, Cano et al, (2019) en Chile, encontraron bajo porcentaje de pacientes con bajo peso con respecto a estudios previos. Los pliegues cutáneos muestran una baja confiabilidad para estimarla masa grasa. La bioimpedanciometría, utilizando la ecuación de Kyle, podría ser un buen método de campo para la evaluación de pacientes en hemodiálisis. Habría que decir también que Dalas y Sanz (2012) en Cuba encontraron que la capacidad funcional y el estado nutricional se asociaron significativamente, la mortalidad del neurópata en 
diálisis crónica al cierre de la ventana de observación fue dependiente de la capacidad funcional: los enfermos fallecidos se destacaron por la limitación de la capacidad funcional. Asimismo, Janardhan et al, (2011) en India, concluyeron que la desnutrición es un índice útil y fiable para la identificación de pacientes en riesgo de desnutrición, ya que correlaciona bien con la evaluación antropométrica y bioquímica. Concepción et al, (2015) en España, concluyeron que existe un grado de desnutrición en pacientes en hemodiálisis y que tras una intervención educativa personalizada se puede corregir los hábitos dietéticos no saludables. Finalmente, Quispe (2014) en Lima, no se encontró ningún paciente con una alimentación adecuada. Concluyeron que una alimentación inadecuada y regular, en este grupo de pacientes, pueden estar ocasionando desnutrición energética y proteica a distintos niveles.

Los métodos de evaluación nutricional son muy importantes y son utilizados en pacientes con riesgo de desnutrición o en desnutrición y cuando sea necesario para hacer indicaciones nutricionales precisas con el objetivo de corregir alteraciones originadas por malnutrición. Se lleva a cabo mediante la aplicación de indicadores de manejo simple y práctico, como los clínicos (pruebas de laboratorio), y antropométricos (pliegues cutáneos) (Ravasco, Anderson, \& Mardones, 2010). Asimismo, para valoración el estado nutricional del paciente con IRC se utiliza dos herramientas: la Valoración Global Subjetiva (VGS) y la Valoración Global Objetiva (VGO).

Respecto a la IRC, esta condición puede tener grados diversos desde fases incipientes o moderadas sin necesidad de terapia de diálisis, hasta la fase avanzada. Se observan manifestaciones clínicas de magnitud variable que incluyen la retención de líquidos, alteraciones electrolíticas y retención de úrea, creatinina y ácido úrico, además de alteraciones endocrinas, inmunológicas, de coagulación, óseas, entre otros. Además, entre más tiempo transcurra, mayor es la probabilidad de mayor mortalidad en la población (Soriano, 2009; Cecil, 2009).

Por lo que se ha observado en la mayoría de los pacientes afiliados, al seguro que ingresan a hemodiálisis debido a la IRC, llegan con un estado nutricional deficiente, con problemas asociados a la insuficiencia renal, socioeconómicos y de salud mental como la resistencia a la enfermedad y depresión, así también problemas gastrointestinales y hábitos alimentarios que finalmente se reflejan en el estado nutricional del paciente, siendo un indicador para un adecuado tratamiento de diálisis y calidad de vida.
Debido a que el Centro de Hemodiálisis SERSALUD Amazonia está comprometido con la calidad de vida de sus pacientes, se busca con esta evaluación la detección de la presencia o riesgo de desnutrición en pacientes en hemodiálisis ambulatoria, para un adecuado manejo nutricional.

Por lo expuesto anteriormente, este estudio tiene el propósito de evaluar el estado nutricional de los pacientes en Hemodiálisis del Centro de Hemodiálisis en la ciudad de Iquitos.

\section{MATERIALES Y MÉTODOS}

El estudio es de enfoque cuantitativo, de diseño no experimental, de corte transversal y de tipo descriptivo (Hernández et al. 2010).

La muestra estuvo conformada por 100 pacientes entre las edades de 20 y 80 años, el $60 \%$ fueron hombres y 40\% mujeres, del Centro de Hemodiálisis SERSALUD Amazonia E.I.R.L. de la ciudad de Iquitos.

Los criterios de inclusión y exclusión fueron: Se incluyeron pacientes en hemodiálisis, de ambos géneros, que acuden a los centros de Hemodiálisis SerSalud y que hayan firmado el consentimiento informado del estudio. Se excluyeron a pacientes que realicen tratamiento de hemodialisis en otro centro, pacientes con algún trastorno o alteración mental y que no hayan firmado el consentimiento informado.

Por otro lado, el instrumento que se utilizó fue la Valoración Global Subjetiva (VGS) y la Valoración Global Objetiva (VGO), que es un método clínico basado en la interpretación clínica y en algunos síntomas y parámetros físicos. Este instrumento de valoración fue tomado del Formato de Screni para pacientes renales con diálisis (Riella et al. 2003).

Siendo el proceso de recolección de datos, previa autorización, se realizó la evaluación de los participantes considerando los siguientes indicadores: antropometría (peso, talla, circunferencia braquial, pliegue cutáneo tricipital), por datos bioquímicos (albúmina, transferrina y recuento de linfocitos totales), por signos clínicos (edemas, ascitis) y dietético (frecuencia de alimentos y recordatorio de 24 horas), y para valorar el diagnóstico según el formato de VGS y VGO.

Los datos fueron analizados mediante el programa SPSS versión 24.0 para Windowns y Excel 2014. Para describir los resultados se hizo mediante tablas de frecuencia y porcentaje. 
Cabe mencionar que este estudio no empleó métodos invasivos, de intervención o modificación intencionada de las variables biológicas, fisiológicas, psicológicas o sociales de los individuos participantes, por lo tanto, se considera una investigación sin riesgo. Se obtendrá el consentimiento informado por escrito de los participantes.

\section{RESULTADOS}

Tabla 1

Distribución de la muestra según la prevalencia de malnutrición calórica proteica

\begin{tabular}{lll}
\hline Variable & $\mathrm{n}$ & $\%$ \\
\hline Índice de masa corporal & 3 & 3.0 \\
$\quad$ Obesidad & 54 & 54.0 \\
$\quad$ Normal & 19 & 19.0 \\
$\quad$ Desnutrición leve & 19 & 19.0 \\
$\quad$ Desnutrición moderada & 5 & 5.0 \\
$\quad$ Desnutrición grave & & \\
Circunferencia Braquial (CB) & 93 & 93.0 \\
$\quad$ Adecuado (100 -90\%) & 7 & 7.0 \\
$\quad$ Desnutrición Leve (90 - 80\%) & & \\
Circunferencia Muscular del Brazo (CMB) & 10 & 10.0 \\
$\quad$ Exceso de peso & 45 & 45.0 \\
$\quad$ Adecuado & 29 & 29.0 \\
$\quad$ Desnutrición leve & 16 & 16.0 \\
$\quad$ Desnutrición moderada & & \\
Pliegue Cutáneo Tricipital (PCT) & & \\
Adecuado & 27.0 \\
Desnutrición leve & & 49.0 \\
Desnutrición moderada & & 24.0 \\
Total & & 100.0 \\
\hline
\end{tabular}

En la Tabla 1 se observa que el 54\% presenta estado nutricional normal por IMC; el 19\%, desnutrición leve y moderada; el 5\%, desnutrición grave; y solo el 3\%, obesidad. Por otro lado, el 93\% tiene una circunferencia braquial adecuada y el $7 \%$ presenta una desnutrición leve, por evaluación del CMB, el $45 \%$ presenta reserva proteica adecuada; el $29 \%$, desnutrición leve; y el 10\%, exceso de peso. En cuanto al pliegue cutáneo tricipital, el $27 \%$ es adecuado; el 49\%, presenta desnutrición leve; y el 24\%, desnutrición moderada.

\section{Tabla 2}

Distribución de la muestra por diagnóstico por parámetros bioquímicos

\begin{tabular}{lll}
\hline Variable & $\mathrm{n}$ & $\%$ \\
\hline Albúmina sérica & & \\
$\quad$ Adecuado $(>4.0 \mathrm{~g} / \mathrm{dl})$ & 18 & 18.0 \\
$\quad$ Riesgo moderado de morbimortalidad $(3.1-4.0 \mathrm{~g} / \mathrm{dl})$ & 76 & 76.0 \\
$\quad$ Alto riesgo de morbimortalidad $(<3.0 \mathrm{~g} / \mathrm{dl})$ & 6 & 6.0 \\
Transferrina sérica & & 5.0 \\
$\quad$ Adecuado $(<180 \mathrm{mg} / \mathrm{dl})$ & 5 & 33.0
\end{tabular}




\begin{tabular}{lll}
\hline Variable & $\mathrm{n}$ & $\%$ \\
\hline \multicolumn{1}{c}{ Desnutrición moderada $(100-150 \mathrm{mg} / \mathrm{dl})$} & 60 & 62.0 \\
Recuento de linfocitos totales & & \\
Adecuado $(>1.500 \mathrm{~mm} 3)$ & 53 & 54.0 \\
Desnutrición leve $(1.201-1.500 \mathrm{~mm} 3)$ & 32 & 31.0 \\
Desnutrición moderada $(800-1.200 \mathrm{~mm} 3)$ & 13 & 13.0 \\
Desnutrición grave $(<800 \mathrm{~mm} 3)$ & 2 & 2.0 \\
Total & 100 & 100.0 \\
\hline
\end{tabular}

En la Tabla 2, según el nivel de albumina sérica, se aprecia que el $76 \%$ tiene un riesgo moderado de mortalidad; y el 6\%, tiene un alto riesgo de morbimortalidad. Asimismo, respecto a la transferrina sérica, el 60\% se encuentra en desnutrición modera- da; y el 5\% presenta niveles adecuados. Finalmente, en cuanto al recuento de linfocitos totales, el 53\% presenta niveles adecuados; el 32\% presenta niveles de desnutrición leve; y el 2\% presenta desnutrición grave.

Tabla 3

Distribución de la muestra por diagnóstico por signos clínicos

\begin{tabular}{lll}
\hline Variable & $\mathrm{n}$ & $\%$ \\
\hline Edema/ascitis & & \\
$\quad$ Ninguna & 81 & 81.0 \\
$\quad$ Leve & 19 & 19.0 \\
Total & 100 & 100.0 \\
\hline
\end{tabular}

En la Tabla 3 se observa que el $81 \%$ no presenta signos de edema o ascitis, y solo el $19 \%$ presenta signos clínicos leves.

Tabla 4

Distribución de la muestra por recordatorio de 24 horas

\begin{tabular}{lll}
\hline Variable & $\mathrm{n}$ & $\%$ \\
\hline Ingesta proteica & & \\
$\quad$ Desnutrición leve $(0.9-1.2 \mathrm{~g} / \mathrm{Kg})$ & 9 & 9.0 \\
$\quad$ Desnutrición moderada $(0.6-0.9 \mathrm{~g} / \mathrm{Kg})$ & 47 & 47.0 \\
$\quad$ Desnutrición grave $(<0.6 \mathrm{~g} / \mathrm{Kg})$ & 44 & 44.0 \\
Ingesta calórica & & \\
$\quad$ Excede & 2 & 2.0 \\
$\quad$ Cumple & 6 & 6.0 \\
$\quad$ Inadecuado (no cumple) & 92 & 92.0 \\
Total & 100 & 100.0 \\
\hline
\end{tabular}

En la Tabla 4 se observa la evaluación dietética por recordatorio de 24 horas donde, por la ingesta proteica, el $47 \%$ presenta desnutrición moderada; el 44\%, desnutrición grave; y 9\%, desnutrición leve.
En cuanto a la ingesta calórica por 24 horas, el 96\% se encuentra inadecuado, el $6 \%$ cumple o es adecuado en su requerimiento y el $2 \%$ se excede en su requerimiento. 


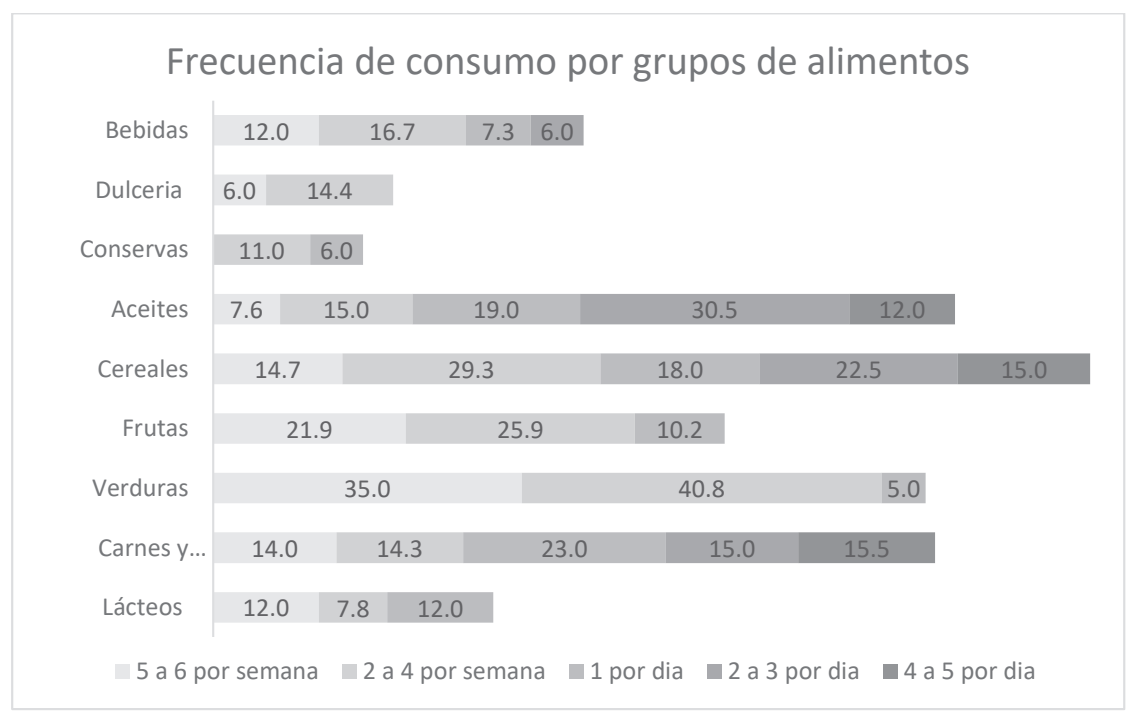

Figura 1. Distribución de la muestra por frecuencia de consumo por grupos de alimentos

Demuestra que la frecuencia de alimentos, por grupos de alimentos, el $40.8 \%$ de los participantes consumen verduras de 2 a 4 porciones por semana; el 25.9\%, frutas; el 29.3\%, cereales. En cuanto al consumo de carnes y sustitutos, el 23\%, consume 1 porción al día; y el 30.5\%, consume aceites de 2 a 3 porciones al día.

Tabla 5

Distribución de la muestra por valoración global subjetiva y objetiva

\begin{tabular}{lll}
\hline Variable & $\mathrm{N}$ & $\%$ \\
\hline Valoración global subjetiva & & \\
$\quad$ Adecuado & 1 & 1.0 \\
$\quad$ Riesgo nutricional/ Desnutrición leve & 99 & 99.0 \\
Valoración global objetiva & & \\
$\quad$ Riesgo nutricional/Desnutrición leve & 75 & 75.0 \\
$\quad$ Desnutrición moderada & 25 & 25.0 \\
Total & 100 & 100.0 \\
\hline
\end{tabular}

La Tabla 5 muestra que el 99\% se encuentra en riesgo nutricional o desnutrición leve, y solo el 1\% en un nivel adecuado, referente a la Evaluación global subjetiva. Mientras que, por valoración global objetiva, el $75 \%$ se encuentra en riesgo nutricional o desnutrición leve y el 25\% en desnutrición moderada.

\section{DISCUSIÓN}

Evaluar el estado nutricional es un proceso sistemático que permite obtener, verificar e interpretar datos, que expliquen la causa y el estado de los problemas relacionados con la nutrición de los individuos Martí- nez et al, (2010). Se han propuesto distintos métodos para valorar el estado nutricional en pacientes en HD, como VGS y VGO Yuste et al, (2013), incluyendo diferentes parámetros de evaluación, como antropométricos, bioquímicos, clínicos y dietéticos.

El estudio tuvo como objetivo evaluar el estado nutricional de pacientes en hemodiálisis a través de la valoración de indicadores antropométricos, bioquímicos, clínicos y dietéticos, además de la Prueba de Valoración Global Subjetiva y el de la Valoración Global Objetiva. Los resultados muestran que solo el $1 \%$ tuvo diagnóstico nutricional adecuado y el $99 \%$ en riesgo o desnutrición, según la VGS; mientras que con la VGO el 75\% presentó riesgo nutricional o desnutrición leve y el 25\% desnutrición moderada. Hay que tener en cuenta que la VGS se basa en componentes como el cambio de peso, cambio en la ingesta alimentaria, síntomas gastrointestinales, la capacidad funcional, comorbilidades relacionadas con el estado nutricional y el examen físico (Schneider 2012), siendo esta prueba más usada que la VGO, la cual evalúa indicadores de peso y talla a través de IMC, ingesta proteica del día anterior y exámenes de laboratorio (Albúmina, Transferrina y el Reencuentro total de linfocitos). Estos resultados indican que más del $50 \%$ de la población, con tratamiento en hemodiálisis, tiene algún tipo de desnutrición. Sin embargo, Yanowsky et al. (2015) utilizaron la VGS en pacientes con hemodiálisis y encontraron que el 34.8\% de pacientes estaban bien nutridos, el 40.6\% tenía riesgo de Desgaste Proteico Energético (DPE) moderado y el 24.6\% presentó un DPE severo. Estos resultados confirman los resultados anteriores en que los pacientes con tratamiento de hemodiálisis tienen algún tipo desnutrición. Asimismo, Espahbodi et al. 
(2014) evaluaron también pacientes en hemodiálisis con la VGS, donde el 93\% tuvo desnutrición moderada y leve, y el 2.8\% desnutrición severa, de la misma manera, Janardhan et al. (2011) evaluaron con la VGS, en la India donde el 91\% de los pacientes en hemodiálisis presentaron desnutrición moderada. Por lo contrario, Noralí (2013), utilizando la VGS en una misma población, encontró al 70\% con estado nutricional normal o adecuado y el 30\% con desnutrición leve. Demostrando así que el estado nutricional en los pacientes en hemodiálisis es variante. Dalas y Sanz (2012) obtuvieron resultados en pacientes en hemodiálisis, que, durante dos años de observación, la desnutrición afecta al 48\% de los pacientes. Indicaron que una evaluación oportuna y completa, en estos pacientes, ayudará a llevar mejor el tratamiento con hemodiálisis. Y aunque no se ha encontrado estudios donde reportan resultados de las valoraciones VGS y VGO, a la vez, estas se complementan y son útiles para detectar y evaluar a pacientes en Hemodiálisis, para una mejor intervención nutricional.

Así también, por parámetro antropométrico según IMC, el 54\% está con nutrición adecuada o normal, mientras que el 19\% con desnutrición leve y moderada, el 5\% con desnutrición grave y con obesidad el 3\%, resultados semejantes encontraron Montalvo y Gómez (2007), ellos obtuvieron mediante el IMC que el 45\% presentó estado nutricional adecuado, el 21\% desnutrición y el 33.3\% sobrepeso y obesidad en pacientes en hemodiálisis. A diferencia, Cano et al. (2010) encontraron, en una muestra de pacientes en hemodiálisis, que el $10 \%$ presentaba desnutrición, el $46.7 \%$ normal, $23.3 \%$ sobrepeso y el $20 \%$ obesidad, según indicador de IMC. En este sentido, la evaluación del estado nutricional, a través del IMC, no es muy fiable para un diagnóstico, es necesario en paciente la medición de los otros indicadores como los de bioquímica, clínico y dietética son necesarios para un mejor diagnóstico.

Por evaluación de reserva calórica proteica en CB, el 93\% se encontró adecuado y el 7\% con desnutrición leve. Asimismo, por CMB el $45 \%$ presenta reserva proteica adecuada, el 29\% desnutrición leve y el 10\% exceso de peso, también por evaluación del PCT, el 27\% presenta adecuada reserva calórica, el 49\% en desnutrición leve y en desnutrición moderada el 24\%. Similares resultados encontraron Quispe (2014), donde el 68\% presentó desnutrición leve por PCT y el $48 \%$ presentó desnutrición leve por CB; de la misma manera, Montalvo y Gómez (2007) obtuvieron el $19 \%$ con adecuada reserva proteica y el 34\% con desnutrición leve por CB, por PCT, obtuvieron el 18.3\% con desnutrición leve y el 70\% en adecuada reserva calórica y por CMB el 19\% con adecuada reserva proteica y el 34\% desnutrición leve. García et al. (2000) encontraron también en una población similar, el 70\% con malnutrición proteica (CB) y el 21\% con desnutrición calórica (PCT). Estas mediciones se realizaron para estimar la masa muscular y grasa corporal, como referencia a la reserva calórica proteica que tenga el paciente, indicándonos sus reservas y el estado nutricional frente al tratamiento de hemodiálisis. Estos indicadores de evaluación aclaran el tipo de desnutrición, ya que son específicos en evaluar reserva calórica - proteica. Aunque difiere al IMC, estos son recomendables en estos tipos de pacientes en el diagnóstico por antropometría.

Al evaluar los indicadores bioquímicos, se encontraron resultados similares. Según los niveles de albúmina sérica, el 76\% presentó desnutrición leve y el 6\% desnutrición moderado. De la misma manera, Quispe (2014) encontró que el 84\% presentó desnutrición proteica visceral (desnutrición leve), en contraste con Montalvo y Gómez (2007) encontraron que el 48\% con valores mayores de albúmina y solo el $2 \%$ con niveles inferiores (desnutrición leve). Con respecto a la transferrina sérica, el $60 \%$ se encuentra en desnutrición moderada, y solo un 5\% tiene niveles adecuados. Y, finalmente, se encontró que el 53\% presenta un adecuado nivel de linfocitos totales, el 32\% desnutrición leve y el 2\% desnutrición grave. Cabe resaltar que no se encontraron resultados respecto a la evaluación por la transferrina sérica y recuento de linfocitos totales. Teniendo en cuenta que los métodos bioquímicos son más sensibles que los antropométricos, y que pueden detectar problemas nutricionales en una etapa precoz, sirviendo para evaluar y monitorear el estado nutricional.

La evaluación de signos clínicos, en la presencia de edemas o ascitis, determinó que el $81 \%$ no presenta signos y el 19\% presenta signos clínicos leves. Sabiendo que la presencia de edemas o ascitis puede ser un signo de desnutrición, pero también se puede contribuir a otros problemas como la ineficiencia dialítica o de muchos medicamentos utilizados por los pacientes. En cuanto a la morbilidad de la población (antecedentes patológicos), el 59\% tiene hipertensión arterial y diabetes mellitus a la vez, el 9\% tiene hipertensión arterial y el 24\% tiene solo diabetes mellitus. De la misma manera, Cardoso y Pérez (2015) encontraron que la comorbilidad más frecuente en la hemodiálisis es la diabetes mellitus con $31.5 \%$. Siendo la diabetes mellitus una de las principales causas de muerte en el Perú, y causal principal de la IRC.

En cuanto al diagnóstico por ingesta dietética por recordatorio de 24 horas, se encontró por ingesta proteica, el 47\% presenta desnutrición moderada, el 44\% 
desnutrición grave y 9\% desnutrición leve. En cuanto a la ingesta calórica, el 96\% se encuentra inadecuado, el $6 \%$ cumple o es adecuado en su requerimiento y el $2 \%$ se excede en su requerimiento. Obteniendo los resultados se observa que los participantes del estudio tienen una alimentación inadecuada por recordatorio de 24 horas, y que no cumplen su requerimiento calórico proteico.

En la evaluación de frecuencia de alimentos, muestra el consumo frecuente por grupos de alimentos, donde el $40.8 \%$ de los participantes consumen verduras de 2 a 4 porciones por semana, el 25.9\% frutas, el 29.3\% cereales, así también, el consumo de carnes y sustitutos, el 23\% consume 1 porción al día y el $30.5 \%$ consume aceites de 2 a 3 porciones al día. No cumpliendo con las recomendaciones nutricionales. Determinando que la frecuencia de alimentos, en esta población, es no adecuada y que podría justificarse con esta evaluación los diagnósticos de los demás indicadores. Resultados similares fueron encontraron por Concepción et al. (2015), donde el índice de alimentación saludable era el 37\% inadecuado. Asimismo, Quispe (2014) encontró que el índice de alimentación fue 64\% inadecuada y 36\% regular en una misma población. Encontrando similitud en los estudios de acuerdo a la alimentación y hábitos alimentarios, y que tienen relación con el estado nutricional. Pero estos resultados deberían ser tomado de tres días diferentes, para un mejor diagnóstico dietético, como lo recomienda la literatura.

La prevalencia de malnutrición calórica proteica por valoración de los indicadores antropométricos, según IMC, mostraron que el 54\% presentó un estado nutricional normal, el 19\% con desnutrición leve y moderada, y el 3\% con obesidad, respectivamente. Por CMB el 45\% presenta reserva proteica adecuada, el $29 \%$ desnutrición leve y el $10 \%$ exceso de peso. Mientras, que por el PCT se encontró el 27\% presenta con adecuada reserva calórica, el 49\% con desnutrición leve y el 24\% con desnutrición moderada.
Respecto a los niveles de albúmina, el 76\% con desnutrición leve y el 6\% con alto riesgo, es decir, desnutrición moderada; a diferencia con la transferrina, el $60 \%$ se encontró con desnutrición moderada y el 5\% adecuado. Según el recuento de linfocitos, el 53\% se encontraron adecuado, mientras que el $32 \%$ en desnutrición leve y el $2 \%$ con desnutrición grave o moderada.

Se recomienda evaluar el estado nutricional utilizando ambas valoraciones (global subjetiva y la global objetiva) en pacientes con enfermedades crónicas, para un diagnóstico nutricional completo. Asimismo, crear un instrumento para evaluar frecuencia de alimentos en pacientes en diálisis, teniendo en cuenta el tipo de preparaciones de sus alimentos, tanto para diabéticos e hipertensos, y el recordatorio de 24 horas de tres días diferentes (en diálisis, sin diálisis y fin de semana). Así también, implementar programas de intervención, con el propósito de mejorar los conocimientos y actitudes sobre su alimentación, calidad de vida y estilos de vida. Y finalmente, investigar las variables estilos de vida y calidad de vida en pacientes en hemodiálisis en el Perú.

\section{Declaración de financiamiento y de conflicto de intereses:}

El estudio fue financiado por los autores, quienes declaran no tener algún tipo de conflicto de interés en la investigación realizada.

\section{Correspondencia:}

Bach. Mercy Lizeth Becerra Ortiz

Coordinadora de la Unidad de Nutrición y Dietas. Clínica Adventista Ana Stahl. Av. La Marina N²85, Iquitos e-mail: mercy.becerra@clinicaanastahl.org.pe mrcybeor@gmail.com

\section{REFERENCIAS BIBLIOGRÁFICAS}

Bravo Ramírez AM, Ramos CA, y Hurtado Torres GF. (2010). Composición corporal en pacientes con insuficiencia renal crónica y hemodiálisis. Nutr Hosp.;25(2):245-9.

Cano M, Camousseigt J, Carrasco F, Rojas P, Inostroza J, Pardo A, et al. (2010) Evaluación de la composición corporal en pacientes con insuficiencia renal crónica. Nutr Hosp. 25(4):682-7.

Cardoso CA, Pérez MC. (2015). Estado nutricional y sobrevida de los pacientes dializados en el Hospital Nacional Almazor Aguinaga Asenjo. Marzo-Diciembre 2012. Vol. 53, Journal of Chemical Information and
Modeling. Universidad Católica Santo Toribio de Mogrovejo.

Concepción M, Feijoo P, Martínez LQ, Pérez AB, Antonio I, Egusquiza R, et al. (2015). Valoración del estado nutricional y consumo alimentario de los pacientes en terapia renal sustitutiva mediante hemodiálisis. Enferm Nefrol [Internet]. 18(2):10311. Available from: http://scielo.isciii.es/pdf/enefro/ v18n2/original4.pdf

Dalas Guiber M, Sanz Guzmán DM. (2012). Evaluación Nutricional y Capacidad funcional de pacientes en 
el Servicio de Hemodialisis del Hospital Hermanos Ameijeiras. Memorias Conv Int Salud Pública.

Espahbodi F, Khoddad T, Esmaeili L. (2014). Evaluation of malnutrition and its association with biochemical parameters in patients with end stage renal disease undergoing hemodialysis using subjective global assessment. Nephrourol Mon [Internet]. ;6(3):e16385. Available from:http://www.pubmedcentral.nih.gov/ articlerender.fcgi?artid=4090668\&tool=pmcentrez\& rendertype $=$ abstract

Garcia Álavez ML, Arranz Pérez I, Roldán Sánchez MA, Velázquez Coca C, Millán Galante M, Bernal Porcel P. (2000). Valoración del estado nutricional de los pacientes en nuestra unidad de hemodiálisis. Prevalencia de malnutrición;5:27-30.

Guerrero Risco A. (1999). Nutrición y diálisis adecuada en diálisis peritoneal. Enfermería nefrológica [Internet].5:6-17. Available from: http://www.revistaseden.org/files/ rev36_1. pdf\#page $=3$

Hernandez R, Fernandez C, Baptista P.(2010). Metodología de la investigación. México; 613

Janardhan V, Rani Nv, Thennarasu P, Maheswara Reddy Cu, Soundararajan P, Kannan G, et al. (2011). Prediction of malnutrition using modified subjective global assessment-dialysis malnutrition score in patients on hemodialysis. Indian J Pharm Sci.;73(1):38.

Jiménez Jiménez S, Muelas Ortega F, Segura Torres P, José Borrego Utiel F, Gil Cunquero JM, Liébana Cañada A. (2012). Evaluación global subjetiva y escala de malnutrición-inflamación para valorar el estado nutricional de pacientes en diálisis peritoneal con hipoalbuminemia. Enfermería nefrológica;15(2):87-93.

Martínez M. Villar R. Rodríguez MJ. Bellido D, De Luis Roman D, Bellino D, Garcia P. (2010). Valoración nutricional. In: Dietoterapia, Nutricion clinica $y$ metabolismo. p. 69-78.

Montalvo M, Gómez M. (2007). Valoración nutricional de pacientes en hemodiálisis. Renut. 1(3):66-71.

Noralí RR. Estado Nutricional de pacientes en tratamiento de hemodiálisis. Tesis para obtener el titulo de licenciada en nutrición. Vol. 1, Universidad Abierta Interamericana. 2013.

Cecil Med. N T-R. (2009). Treatment of irreversible renal failure. In: Cecil Medi. Philadelphia: Panamericana: Saunders Elsevier; p. 133.
Palacios Guillen A, Medina Santander B, Campos Buleje S, Berríos Medina E, Solís Vásquez G, Bravo Zúñiga J, et al. Guía clínica para identificación, evaluación y manejo inicial del paciente con enfermedad. Soc Peru Nefrol. 2010;1.

Quispe M V. (2014). Indice de alimentación saludable y el estado nutricional de los pacientes ambulatorios que inician hemodialisis en el Hospital Nacional Dos de Mayo, Lima 2013. Universidad Nacional Mayor de San Marcos.

Ravasco P, Anderson H, Mardones F. (2010). Métodos de valoración del estado nutricional. Nutr Hosp.;25(Supl. 3):57-66.

Riella MC, Martins C. (2003). Nutrición y riñón [Internet]. Panamerica. Nutrición y riñón. 2003. 450 p. Available from: https://books.google.com/ books?id=1CWvtBlNiYoC\&pgis=1

Schneider Meireles M, Catarina A, Castro D, Leister Rocha M, Ayako Kamimura M, Cuppari L.(2012). Description of the Subjective Global Assessment Components in Nondialysis-Dependent Chronic Kidney Disease Patients. Kidney Res Clin Pract [Internet]. 31(2):A27. Available from: http://linkinghub.elsevier.com/retrieve/ pii/S2211913212004020

Secretaria de Salud. Norma Oficial Mexicana NOM-043SSA2 (2013). Servicios básicos de salud. Promoción y educación para la salud en materia alimentaria. Criterios para brindar orientación. D Of la Fed;24(1):24-79.

Sociedad Española de Nefrologia. Informe de diálisis y trasplante (2015). In: XLV Congreso Nacional de la Sociedad Española de Nefrologia RA de ER.. p. 73.

Soriano S HL.(2009). Nefrología Clínica. In: Procedimiento disgnóstico y valoración del enfermo con insuficiencia renal crónica. Editorial . España. p. 801-4.

Yanowsky-escatell FG, Pazarín-villaseñor L, Andradesierra J, Zambrano-velarde MÁ, Preciado-figueroa FM, Jesús C, et al. (2015). Asociación de albúmina sérica y valoración global subjetiva en pacientes incidentes en diálisis peritoneal. Nutr Hosp;32(6):2887-92.

Yuste Claudia, Abad Soraya, Vega Almudena, Barraca Daniel, Bucalo Laura, Pérez de José Ana LGJ. (2013). Valoración del estado nutricional en pacientes en hemodiálisis. Rev Nefrol. ;33(3):462-9.

Recibido: 28/09/16 Aceptado: 06/11/16 